Dieses Dokument ist eine Zweitveröffentlichung (Postprint) /

This is a self-archiving document (postprint):

Mandy Korzetz, Romina Kühn, Uwe Aßmann, Thomas Schlegel

\title{
A Design Kit for Mobile Device-Based Interaction Techniques
}

Erstveröffentlichung in / First published in:

HCII: International Conference on Human-Computer Interaction, Copenhagen 2020. Cham:

Springer, S. 52 -59. ISBN 978-3-030-50726-8

DOI: https://doi.org/10.1007/978-3-030-50726-8 7

Diese Version ist verfügbar / This version is available on:

https://nbn-resolving.org/urn:nbn:de:bsz:14-qucosa2-713004 


\title{
A Design Kit for Mobile Device-Based Interaction Techniques
}

\author{
Mandy Korzetz ${ }^{1}$, Romina Kühn ${ }^{1}$, Uwe Aßmann ${ }^{1}$, and Thomas Schlegel ${ }^{2}$ \\ 1 TU Dresden, Software Technology Group, Dresden, Germany \\ \{mandy.korzetz, romina.kuehn\}@tu-dresden.de \\ 2 Karlsruhe University of Applied Sciences, Institute of Ubiquitous Mobility Systems, \\ Karlsruhe, Germany \\ thomas.schlegel@hs-karlsruhe.de
}

\begin{abstract}
Beside designing the graphical interface of mobile applications, mobile phones and their built-in sensors enable various possibilities to engage with digital content in a physical, device-based manner that move beyond the screen content. So-called mobile device-based interactions are characterized by device movements and positions as well as user actions in real space. So far, there is only little guidance available for novice designers and developers to ideate and design new solutions for specific individual or collaborative use cases. Hence, the potential for designing mobile-based interactions is seldom fully exploited. To address this issue, we propose a design kit for mobile device-based interaction techniques following a morphological approach. Overall, the kit comprises seven dimensions with several elements that can be easily combined with each other to form an interaction technique by selecting at least one entry of each dimension. The design kit can be used to support designers in exploring novel mobile interaction techniques to specific interaction problems in the ideation phase of the design process but also in the analysis of existing device-based interaction solutions.
\end{abstract}

Keywords: Design kit $\cdot$ design space $\cdot$ device-based interaction $\cdot$ gestures $\cdot$ mobile phone.

\section{Introduction}

Smartphones have become constant companions of our daily life. They are included in our daily activities and support us on the way, for example, with playing our favorite music or podcast, writing shopping lists, planning trips, or taking and sharing photos with others. Usually these functions can be started using touch interactions of the graphical interface. Beside touch screens, common smartphones are equipped with a variety of sensors that can be used to provide alternative ways to interact with the device in a physical, device-based manner. For example, users can share information with other devices by performing a throw movement [18], expand the working area by juxtaposing multiple devices [16], or rate displayed content by ordering multiple devices on a table [10]. Such 
mobile device-based interactions provide an easy and fast way to access specific smartphone functionality. They harness built-in sensors to estimate device movements and positions or in general user actions in real space [9]. As mobile usage often occurs when user's attention is divided among multiple tasks, such interactions enable an intuitive and seamless integration into daily activities. Moreover, they can enhance collaborative scenarios by enabling physical multi-device interaction and facilitate working digitally face-to-face without media breaks.

Although there is a lot of research that proposes device-based interaction solutions for individual, e. g. $[19,13]$, as well as collaborative use cases, e. g. $[5,7,11]$, only little guidance is available to support designers and developers in ideating and designing novel device-based interaction solutions. This is one of the main reasons why only a few device-based interactions have found their way into commercial applications. Early phases for designing interfaces often are characterized by sketching. Resources like templates and stencils (e. g. UI Stencils ${ }^{1}$ ) can support paper work, various software tools (e. g. Adobe $\mathrm{XD}^{2}$, Framer $^{3}$ ) support digital design of mobile interfaces. Reference guides or gesture cards (e. g. Touch Reference Guide by Luke Wroblewski ${ }^{4}$ ) help designing touch interactions. Such tools focus on the visual aspects of designing interfaces but lack supporting physical interaction and rely on knowledge of the possibilities of the device sensors that go beyond the screen. Hence, the potential for designing mobile interactions is seldom fully exploited. To fill this gap, we propose a design kit to support designers and developers of mobile interfaces that is suited for device-based interaction techniques. Furthermore, the kit enables designers and developers to get a general idea of physical interaction possibilities.

Our design kit follows a morphological approach developed by Zwicky [21] and intends to support especially the early phases of designing interactions. It can be used twofold: for exploring and communicating novel interaction techniques during ideation (bottom-up approach) but also for analyzing existing device-based interaction solutions to learn from them (top-down approach). The kit comprises seven dimensions with different elements that can be easily combined with each other to form an interaction technique. The dimensions span the design space for physical interaction techniques with mobile phones, and help researchers and practitioners to continue working on concepts that enhance mobile interaction.

The paper is organized as follows: First, we give an overview of related work concerning approaches for designing interactions and gestures as well as using morphology for design. Based on this, we describe the details of our design kit for mobile device-based interaction techniques and how it can be applied in interaction design. Finally, we discuss the implications of this work and give an outlook on next steps.

\footnotetext{
1 https://www.uistencils.com/

${ }^{2}$ https://www.adobe.com/at/products/xd.html

3 https://www.framer.com/

${ }^{4}$ https://static.lukew.com/TouchGestureGuide.pdf
} 


\section{Related Work}

In order to develop a design kit to define novel concepts for mobile devicebased interactions, we considered approaches for designing interactions within the related research area of gestural interfaces. Furthermore, we examined related work in terms of using the morphological approach for design.

\subsection{Approaches for Gestural Interaction Design}

Nielsen et al. proposed a participatory design approach by involving potential end users in the design process [17]. The design process works as follows: given several tasks, study participants were asked to specify gestures that would execute these tasks. Commonly used gestures were collected in a gesture set. Within a last step, the resulting gesture set is checked back by asking participants to guess functions to presented gestures. Designing gestures with user involvement has been applied in different interaction domains, e. g. hand gestures [17], surface gestures for tabletop interaction [20], motion gestures with mobile phones [19], and three-dimensional mobile gestures for smart home control [12]. The participatory approach is intended to reach a high level of intuitiveness and ergonomics in the developed gestures but is also a complex and time consuming process. Additionally, it needs knowledge about user research methods. Because of the high effort it is less appropriate for projects with limited time and budget. Furthermore, mobile-based interactions are still relatively unfamiliar to users, the outcome of novel ideas may be limited. Overall, existing interaction solutions are only taken into consideration to a very limited extent. To meet the requirements of the user's needs as well as technological innovations, interaction designers should start with their ideas from metaphors [6], which we included in our design kit as first inspiration.

\subsection{Morphology for Design}

Using a morphological box for solving problems can be traced back to Zwicky, who introduced it to the classification of astrophysical objects and the design of jet engines and rocket propulsion systems [21]. It is a methodical framework for structuring and analysing any type of multi-dimensional, non-quantified problem with the aim to explore possible solutions [1]. As a morphological box maps all possible parameters of solutions, it also defines the design space of an abstract construct. The morphological approach has been applied to many diverse domains, from engineering and product design to policy analysis and organisational design. In the fields of visualization and interfaces, the morphological approach was applied, for example, to the graphical design of diagrams, networks, and maps [2] and to timeline design [3]. Mackinlay and Card proposed the morphological approach for describing the design space of input devices [14,4]. To our best knowledge there is no research that applied that morphology to physical interaction with mobile phones. To address this issue, we propose a design kit especially for mobile device-based interaction techniques and define the design space to support producing novel interaction designs. 
Final edited form was published in "HCII: International Conference on Human-Computer Interaction. Copenhagen 2020",

S. 52 -59. ISBN: 978-3-030-50726-8

https://doi.org/10.1007/978-3-030-50726-8_7

\section{Interaction Design Kit}

The development of our design kit bases on a comprehensive analysis of related work from research and practice, which includes a broad range of specific interaction solutions for individual (e. g. $[13,19,18]$ ) and collaborative usage scenarios with multiple devices (e. g. $[5,7,10,11,16])$ as well as the mobile spaces model by Korzetz et al. [9]. We extracted relevant elements and aggregated them to dimensions using reverse engineering. The elements were combined with each other to form an interaction technique. Figure 1 shows the design kit with its seven dimensions. In the following, we address these dimensions in more detail.

\section{ACTION}

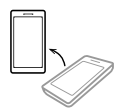

Lift vs.

Put down

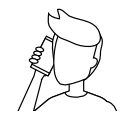

Place to body

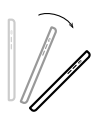

Swing
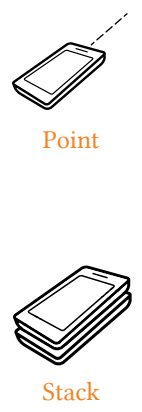

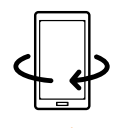

Tilt

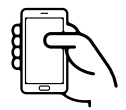

Squeeze
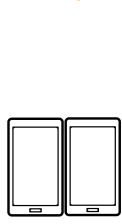

Juxtapose

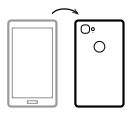

Flip

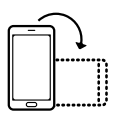

Orientate

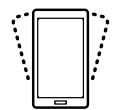

Shake

\section{MOBILE SENSORS}

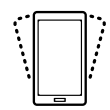

Motion

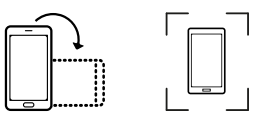

Orientation Environment

\section{Position}

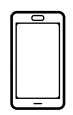

Upright vs, Downwards
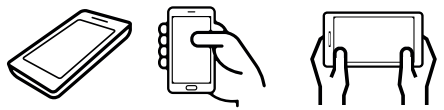

$\frac{\text { w/o Hands }}{\text { Lying }}$

$\frac{\text { One-handed Two-handed }}{\text { Holding }}$

Coupling Style

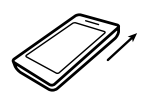

Shift vertically
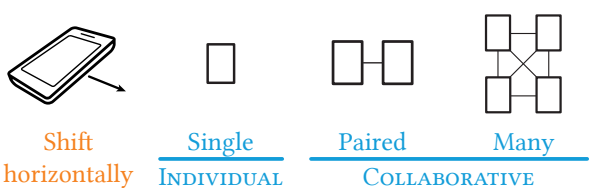

\section{ArRangemient}

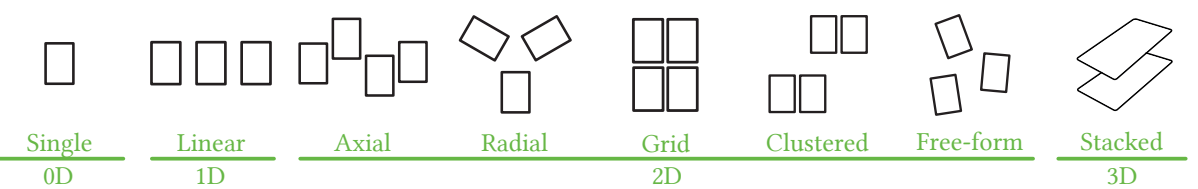

\section{MOVEMENT}

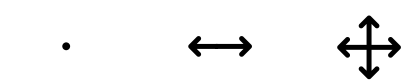

$\frac{\text { None }}{\text { StATIC }}$

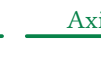

Flat

DyNAMIC

\section{Distribution}
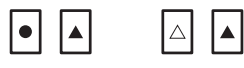

Distinct Transferred
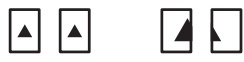

Spatial

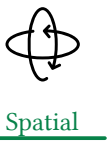

Distinct
Mirrored
Composed

Fig. 1. Overview of the design kit with its elements within the seven dimensions. 
Final edited form was published in "HCII: International Conference on Human-Computer Interaction. Copenhagen 2020",

S. 52 -59. ISBN: 978-3-030-50726-8

https://doi.org/10.1007/978-3-030-50726-8_7

Action. This dimension describes the users' possibilities to manipulate their devices physically, e. g. flipping [19] or swinging [18]. The elements of this dimension cover user actions which are simple and lightweight to use and well-known from everyday life.

Position. The position describes the placement of the device in relation to the users' hands. Users can hold the phone with one hand or with both hands. It is also possible to design an interaction where the device is lying on a surface.

Coupling style. This dimension characterizes the devices' connection within a device group (cp. [15]). Devices can operate independently (single), in pairs (paired), or each device is connected to all others (many).

Arrangement. This dimension refers to the physical arrangement of one or more mobile devices. An arrangement can be static or serve as a starting point for dynamic interactions where devices are replaced.

Movement. The movement dimension maps possible device motions in space that range from a static position (none) to dynamic motions in one (axis), two (flat), or three dimensions (spatial).

Distribution. This dimension describes how information is distributed and if it is available for all devices at any time. Information can be transferred from one device to another, mirrored to other devices, or information among devices is composed or distinct.

Mobile sensors. Mobile sensors bridge the gap between design and implementation. This dimension refers to the main sensor classes of motion (e. g. accelerometer), orientation, and environmental sensors (e. g. illumination).

Inspirational metaphors. An additional set of metaphors (see Fig. 2) that we excerpted from our literature review helps designers to organize design thinking and to identify appropriate elements for their linked system functions.

\section{INSPIRATIONAL METAPHORS}

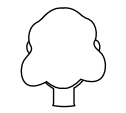

Face away

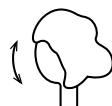

Nod vs. Shake head

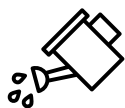

Pour

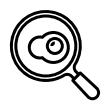

Pan

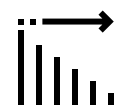

Order

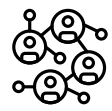

Group

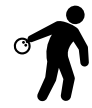

Throw vs.

Fetch

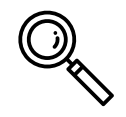

Lens

Fig. 2. Metaphors as additional ideation support (extract). 


\section{Usage of the Design Kit}

Our design kit can be applied bottom-up for creating and exploring novel interaction solutions (composing) but also in a top-down approach for analyzing existing solutions by decomposing interaction techniques. For composing new interaction techniques, the elements can be combined by assigning at least one element from each of the seven dimensions. Figure 3 shows Order to Vote [10] as an example of a mobile-based interaction technique, where multiple devices are arranged in a row on a table and users are enabled to rate displayed content by rearranging their order. This interaction can be described by combining the elements juxtapose, lying, many coupling, linear arrangement, axial movement, distinct content distribution, and by using the motion sensors. In further steps, designers can complement the resulting combination by describing the connected task (here: voting or rating) and how the connection works (discrete or continuous). The main application of the design kit is exploring new and different mobile-based interactions to facilitate brainstorming and communicating interaction ideas. This can be done by using the provided elements. Moreover, it serves as a starting point for capturing interaction knowledge by extending the design kit with new elements if possible.

\section{Conclusion and Future Work}

We proposed a design kit for mobile device-based interaction techniques, which are characterized by physical manipulations in real space. The design kit aims at supporting designers and developers in the ideation phase of the design process. It follows a morphological approach and therefore provides a structured inventory of possible solutions. The kit comprising of dimensions and elements can be easily applied top-down for analyzing existing interaction solutions and bottomup for exploring and composing novel interaction techniques. With an example, we presented how users can capture their ideas by combining the elements. To ensure the reuse of proven solutions, we plan to expand the capturing in form of interaction patterns.

Our next steps include a user study with designers and developers of mobile interfaces to assess the structure and elements of the design kit in terms of the usage and completeness. We further plan a digital variant of our current paper-based design kit that enables users to integrate their interactions in existing design tools. To support further processing in terms of creating interactive prototypes, we intend to integrate relevant kit elements in our MilkyWay

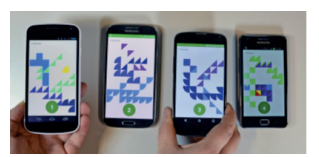

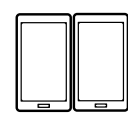

Juxtapose

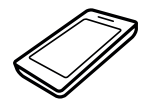

Lying

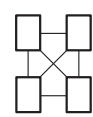

Many

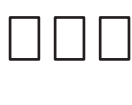

Linear

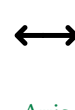

Axis

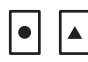

Distinct

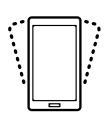

Motion

Fig. 3. Description of Order to Vote [10] with the design kit. 
toolbox [8]. As mobile device-based interaction solutions exist in research, we hereby provide an easy to use tool with the aim to gain more visibility of mobile device-based interactions in practice.

\section{Acknowledgements}

The European Social Fund (ESF) and the German Federal State of Saxony have funded this work within the project CyPhyMan (100268299). Also funded by the German Research Foundation (DFG, Deutsche Forschungsgemeinschaft) as part of Germany's Excellence Strategy - EXC 2050/1 - Project ID 390696704 - Cluster of Excellence "Centre for Tactile Internet with Human-in-the-Loop" (CeTI) of Technische Universität Dresden. The figures of this work include icons made by Flaticon (https://www.flaticon.com).

\section{References}

1. Álvarez, A., Ritchey, R.: Applications of General Morphological Analysis: From Engineering Design to Policy Analysis 4(1) (2015)

2. Bertin, J.: Semiology of graphics. University of Wisconsin Press (1983)

3. Brehmer, M., Lee, B., Bach, B., Riche, N.H., Munzner, T.: Timelines revisited: A design space and considerations for expressive storytelling. IEEE Transactions on Visualization and Computer Graphics 23(9), 2151-2164 (2017)

4. Card, S.K., Mackinlay, J.D., Robertson, G.G.: A morphological analysis of the design space of input devices. ACM Transation on Information Systems 9(2), 99-122 (1991). https://doi.org/10.1145/123078.128726

5. Jokela, T., Lucero, A.: FlexiGroups: binding mobile devices for collaborative interactions in medium-sized groups with device touch. In: Quigley, A., Diamond, S., Irani, P., Subramanian, S. (eds.) Proceedings of the 16th ACM International Conference on Human-Computer Interaction with Mobile Devices and Services. pp. 369-378. ACM (2015). https://doi.org/10.1145/2628363.2628376

6. Keck, M., Lapczyna, E., Groh, R.: Revisiting graspable user interfaces. In: Marcus, A. (ed.) Design, User Experience, and Usability. Theories, Methods, and Tools for Designing the User Experience. pp. 130-141. Springer Internat. Publishing (2014)

7. Korzetz, M., Kühn, R., Heisig, P., Schlegel, T.: Natural Collocated Interactions for Merging Results with Mobile Devices. In: Proceedings of the 18th International Conference on Human-Computer Interaction with Mobile Devices and Services Adjunct. pp. 746-752. MobileHCI '16, ACM (2016). https://doi.org/10.1145/2957265.2961839

8. Korzetz, M., Kühn, R., Kegel, K., Georgi, L., Schumann, F.W., Schlegel, T.: Milkyway: A toolbox for prototyping collaborative mobile-based interaction techniques. In: Antona, M., Stephanidis, C. (eds.) Universal Access in Human-Computer Interaction. Multimodality and Assistive Environments. pp. 477-490. Springer International Publishing, Cham (2019). https://doi.org/10.1007/978-3-030-23563-5_38

9. Korzetz, M., Kühn, R., Schlegel, T.: Turn it, Pour it, Twist it: A Model for Designing Mobile Device-Based Interactions. In: Proceedings of the 5th International Conference on Human-Computer Interaction and User Experience in Indonesia. pp. 20-23. CHIuXiD '19, ACM (2019). https://doi.org/10.1145/3328243.3328246 
10. Kühn, R., Korzetz, M., Büschel, L., Korger, C., Manja, P., Schlegel, T.: Natural Voting Interactions for Collaborative Work with Mobile Devices. In: Proc. of the CHI Conference Extended Abstracts on Human Factors in Computing Systems. pp. 2570-2575. CHI EA '16, ACM (2016). https://doi.org/10.1145/2851581.2892300

11. Kühn, R., Korzetz, M., Schumann, F.W., Büschel, L., Schlegel, T.: Vote-for-it: Investigating mobile device-based interaction techniques for collocated anonymous voting and rating. In: Lamas, D., Loizides, F., Nacke, L., Petrie, H., Winckler, M., Zaphiris, P. (eds.) Human-Computer Interaction - INTERACT 2019. pp. 585-605. Springer (2019). https://doi.org/10.1007/978-3-030-29381-9_36

12. Kühnel, C., Westermann, T., Hemmert, F., Kratz, S., Müller, A., Möller, S.: I'm home: Defining and evaluating a gesture set for smart-home control. International Journal of Human-Computer Studies 69(11), 693-704 (2011). https://doi.org/10.1016/j.ijhcs.2011.04.005

13. Ljubic, S., Kukec, M., Glavinic, V.: Tilt-Based Support for Multimodal Text Entry on Touchscreen Smartphones: Using Pitch and Roll. In: Stephanidis C., A.M.e. (ed.) Universal Access in Human-Computer Interaction. Applications and Services for Quality of Life, Lecture Notes in Computer Science, vol. 8011, pp. 651-660. Springer Berlin Heidelberg (2013). https://doi.org/10.1007/978-3-642-39194-1_75

14. Mackinlay, J., Card, S.K., Robertson, G.G.: A semantic analysis of the design space of input devices. Human-Computer Interaction 5(2-3), 145-190 (1990). https://doi.org/10.1080/07370024.1990.9667153

15. Neumayr, T., Jetter, H.C., Augstein, M., Friedl, J., Luger, T.: Domino: A Descriptive Framework for Hybrid Collaboration and Coupling Styles in Partially Distributed Teams. Proceedings of the ACM on Human-Computer Interaction 2(CSCW), 1-24 (2018). https://doi.org/10.1145/3274397

16. Nielsen, H.S., Olsen, M.P., Skov, M.B., Kjeldskov, J.: Juxtapinch: Exploring multidevice interaction in collocated photo sharing. In: Proceedings of the 16th International Conference on Human-Computer Interaction with Mobile Devices \& Services. p. 183-192. MobileHCI '14, Association for Computing Machinery (2014). https://doi.org/10.1145/2628363.2628369

17. Nielsen, M., Störring, M., Moeslund, T.B., Granum, E.: A procedure for developing intuitive and ergonomic gesture interfaces for hci. In: Camurri, A., Volpe, G. (eds.) Gesture-Based Communication in Human-Computer Interaction. pp. 409420. Springer (2004). https://doi.org/10.1007/978-3-540-24598-8_38

18. Paay, J., Raptis, D., Kjeldskov, J., Lauridsen, B.M., Penchev, I.S., Ringhauge, E., Ruder, E.V.: A comparison of techniques for cross-device interaction from mobile devices to large displays. In: Proceedings of the 14th International Conference on Advances in Mobile Computing and Multi Media. p. 137-146. MoMM '16, ACM (2016). https://doi.org/10.1145/3007120.3007140

19. Ruiz, J., Li, Y., Lank, E.: User-defined motion gestures for mobile interaction. In: Tan, D. (ed.) Proceedings of the SIGCHI Conference on Human Factors in Computing Systems (CHI '11). p. 197. ACM Digital Library, ACM, New York, NY (2011). https://doi.org/10.1145/1978942.1978971

20. Wobbrock, J.O., Morris, M.R., Wilson, A.D.: User-defined gestures for surface computing. In: Proceedings of the SIGCHI Conference on $\mathrm{Hu}-$ man Factors in Computing Systems. p. 1083-1092. CHI '09, ACM (2009). https://doi.org/10.1145/1518701.1518866

21. Zwicky, F.: The morphological approach to discovery, invention, research and construction. In: Zwicky, F., Wilson, A.G. (eds.) New Methods of Thought and Procedure. pp. 273-297. Springer Berlin Heidelberg, Berlin, Heidelberg (1967) 